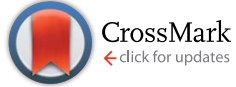

Cite this: J. Mater. Chem. A, 2016, 4, 12774

Received 22nd April 2016

Accepted 14th July 2016

DOI: $10.1039 / c 6 t a 03364 d$

www.rsc.org/MaterialsA

\section{Conducting Al and Ga-doped zinc oxides; rapid optimisation and scale-up †}

\begin{abstract}
D. P. Howard, P. Marchand, I. D. Johnson, C. J. Carmalt, I. P. Parkin and J. A. Darr*
A high-throughput synthesis, screening and subsequent scale-up approach was utilised for the optimisation of conductive aluminium and gallium-doped zinc oxide (AZO and GZO, respectively) nanoparticles. AZO and GZO nanoparticles with up to 6 at\% dopant (with respect to $\mathrm{Zn}$ ) were directly synthesised using a laboratory scale continuous hydrothermal process at a rate of $60 \mathrm{~g}$ per hour. The resistivities were determined by Hall effect measurements on pressed, heat-treated discs. Both Al- and Ga-doping yielded resistivities of the order of $1 \times 10^{-2} \Omega \mathrm{cm}$ for most samples; the lowest resistivity of AZO was $7.0 \times 10^{-3}$ $\Omega \mathrm{cm}$ (at 2.5 at\% Al doping), and the lowest resistivity of GZO was $9.1 \times 10^{-3} \Omega \mathrm{cm}$ (at 3.5 at\% Ga doping), which are considered exceptionally conductive for pressed nanopowders. Synthesis of the optimised lab-scale compositions was scaled-up using a pilot-scale continuous hydrothermal process at a production rate of $8 \mathrm{~kg}$ per day (by dry mass); results obtained from these nanopowders generally retained resistivity trends observed for the lab-scale analogues.
\end{abstract}

\section{Introduction}

Transparent Conducting Oxides (TCOs) are among the most important classes of advanced functional materials in widespread use today, due to the combination of high optical transparency and low electrical resistivity that are fundamental to their use. ${ }^{\mathbf{1 , 2}}$ These properties are necessary in the development of applications such as touchscreens, ${ }^{3}$ solar cells, ${ }^{2,4}$ smart windows, ${ }^{5}$ organic light emitting diodes (OLEDs) ${ }^{6}$ and flat panel displays. $^{7}$ In order to achieve high transparency and conductivity within the materials, the introduction of nonstoichiometry with respect to oxygen is often required, which can be substituted by or coupled with the introduction of aliovalent dopants into the wide band gap $(>3 \mathrm{eV})$ semiconductor, depending on the material in question. ${ }^{8}$ With low resistivity (typically $10^{-4} \Omega \mathrm{cm}$ ) and high optical transparency $>80 \%$ for thin films, tin-doped indium oxide (ITO) is the industry standard material.1,9 Given the increasing scarcity and cost of indium, alternative materials for TCOs are under investigation, focussing on materials such as doped zinc oxides, and titanium and tin dioxides. ${ }^{\mathbf{1 0 , 1 1}}$ In particular, two promising TCO materials are aluminium-doped zinc oxide (AZO) and gallium-doped zinc oxide (GZO), both of which have been shown to exhibit conductivity and optical transparency characteristics rivalling those of ITO. $^{\mathbf{1 2}}$ Highly promising optoelectronic properties aside, in terms of abundance and cost, zinc oxide-based

Department of Chemistry, University College London, 20 Gordon Street, London WC1H oAJ, UK. E-mail: j.a.darr@ucl.ac.uk

$\dagger$ Electronic supplementary information (ESI) available. See DOI: 10.1039/c6ta03364d alternatives are among the foremost potential replacement TCO materials. Dense thin films of such materials can be deposited using a wide range of techniques including sputtering, ${ }^{13}$ chemical vapour deposition, ${ }^{\mathbf{1 4}}$ pulsed electron deposition, ${ }^{\mathbf{1 5}}$ and pulsed laser deposition. ${ }^{\mathbf{1 6}}$ Some of these processes can require annealing steps in excess of $500{ }^{\circ} \mathrm{C}$ in order to obtain optimal performance. ${ }^{9}$ This would restrict the choice of substrate to those that can withstand such conditions, limiting the applicability of TCO material deposition onto some plastics for applications in flexible displays, for example. The use of highly crystalline TCO materials, formulated into inks, pastes, or dispersions, would allow them to be deposited onto a wider range of substrates using techniques such as ink-jet or screenprinting processes. ${ }^{17-19}$ Coupled with low temperature, relatively mild post-deposition processes, large-scale manufacturing of printed optoelectronics becomes possible. ${ }^{20}$

The synthesis of transparent conducting nanomaterials includes methods such as batch coprecipitation and batch solvothermal and hydrothermal methods. ${ }^{17,21-24}$ However, the use of organic solvents with high volatile organic compound (VOC) content is unsustainable and batch-to-batch variations can make these methods unsuitable for industrial scale-up. ${ }^{17,25,26}$

Continuous Hydrothermal Flow Synthesis (CHFS) processes offer the possibility of controlled and consistent syntheses of nanomaterials at large scale. In such processes, nanoparticles of metal oxides are typically formed upon mixing a feed of supercritical (sc) water with a stream of ambient-temperature aqueous metal salts. The precursors react with the sc-water resulting in simultaneous hydrolysis and dehydration of the metal salt to the corresponding metal oxide, typically in less than a second. In recent years, the use of a Confined Jet Mixer 
(CJM) has been reported by the authors for the continuous synthesis of various nanoparticles. In the CJM, a flow of aqueous precursors is entrained in a jet of supercritical water, affording rapid mixing, which is generally under turbulent conditions. ${ }^{27-29}$ The CJM is also highly scalable, such that similar particle properties have been observed for nanoparticles made on the lab and pilot plant scales. ${ }^{30,31}$

For the discovery and optimisation of TCO nanomaterials, the development of high-throughput synthesis and rapid screening methods for doped metal oxide systems is highly desirable. Synthesis of doped metal oxides can readily be accomplished by mixing the appropriate ratios of metal salts (e.g. nitrate salts of the dopant or most metals in the oxide) into a single precursor solution, which is then used in the CHFS process. One particular benefit of CHFS-type reactors is that many different compositions of such doped materials can be synthesised sequentially by simply adjusting the relative ratio of the various metal salt solutions in the precursor feed; great care must be taken to avoid cross-contamination between different samples. ${ }^{31}$ Variations of this high-throughput approach for CHFS have previously been demonstrated by some of the authors for the synthesis of an entire phase diagram of $\mathrm{Ce}_{x} \mathrm{Zr}_{y} \mathrm{Y}_{z} \mathrm{O}_{2-\delta},{ }^{30-33}$ Eu-doped $\mathrm{Y}(\mathrm{OH})_{3}$ phosphor libraries, ${ }^{34}$ Fe-doped $\mathrm{La}_{4} \mathrm{Ni}_{3} \mathrm{O}_{10}$ mixed ion conductor libraries, ${ }^{35}$ and a rare earth-doped zinc oxide photocatalyst library. ${ }^{32}$ Application of this high-throughput approach to TCO systems would be expected to facilitate rapid optimisation of dopant concentrations for maximised conductivity.

The use of CHFS for the direct/indirect synthesis of TCO nanoparticles is limited in the literature; ITO has recently been synthesised via CHFS methods by some of the authors with pressed-disc resistivities as low as $6.0 \times 10^{-3} \Omega \mathrm{cm} \cdot{ }^{31}$ The authors have also investigated the CHFS synthesis of co-doped zinc oxide (with both $\mathrm{Al}$ and Ga and known as AGZO) using formic acid as an in-process reducing agent, which gave a resistivity of $4.6 \times 10^{-2} \Omega \mathrm{cm} .^{36}$

Herein, we report the first CHFS synthesis of highly conductive optimised AZO and GZO nanoparticles followed by heat-treatment in reducing conditions. Compositional space for each doped system was first explored (up to 6 at\%) using a labscale CHFS reactor at a production rate of $60 \mathrm{~g}$ per hour. These materials were screened as heat-treated pellets, and then the composition with the highest conductivity was remade using a pilot-scale CHFS process at a production rate of $8 \mathrm{~kg}$ per day (by dry mass). The conductivity of this material was also evaluated as a heat-treated pellet.

\section{Experimental}

\section{Materials}

Reagents were purchased from the following suppliers and used as-purchased: zinc nitrate hexahydrate, 98\% (Sigma Aldrich, Dorset, UK), aluminium nitrate nonahydrate, 98+\% (Sigma Aldrich, Dorset, UK), gallium nitrate hydrate, 99.999\% (Alfa Aesar, Lancashire, UK), and potassium hydroxide (Fisher Scientific, Leicestershire, UK).

\section{Nanoparticle synthesis by CHFS}

The lab-scale CHFS process was used, similarly to that reported elsewhere. ${ }^{37,38}$ In the lab-scale CHFS, three identical diaphragm pumps (Primeroyal K, Milton Roy, Pont Saint-Pierre, France) were used to supply three pressurised feeds at 24.1 MPa as follows; (i) DI water at a flow rate of $80 \mathrm{~mL} \mathrm{~min}^{-1}$ (pump $\mathrm{P}_{1}$ ), (ii)

\section{a) CONTINUOUS HYDROTHERMAL FLOW SYNTHESIS}

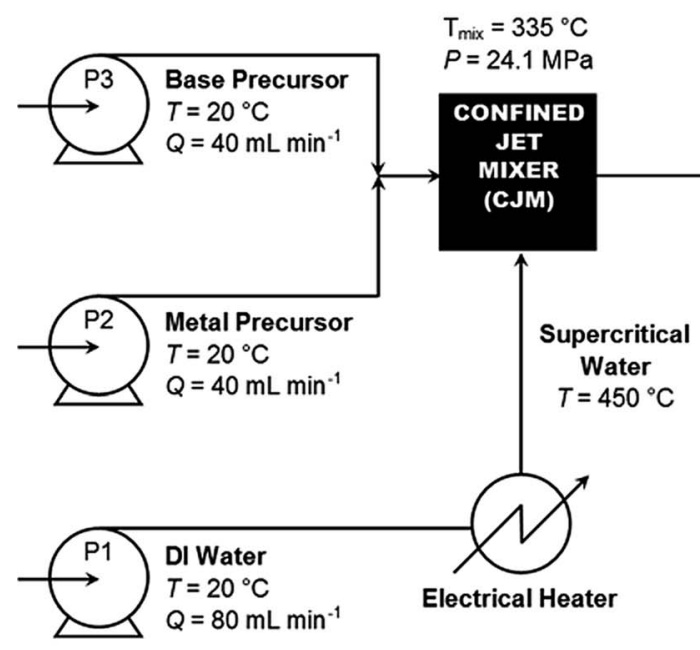

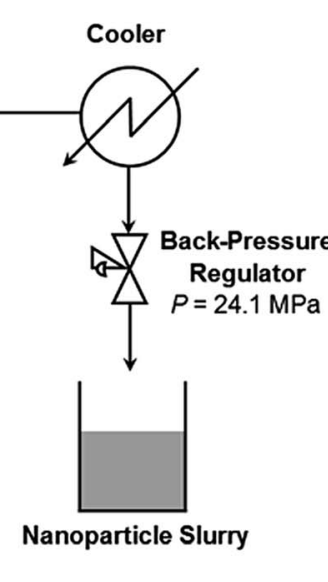

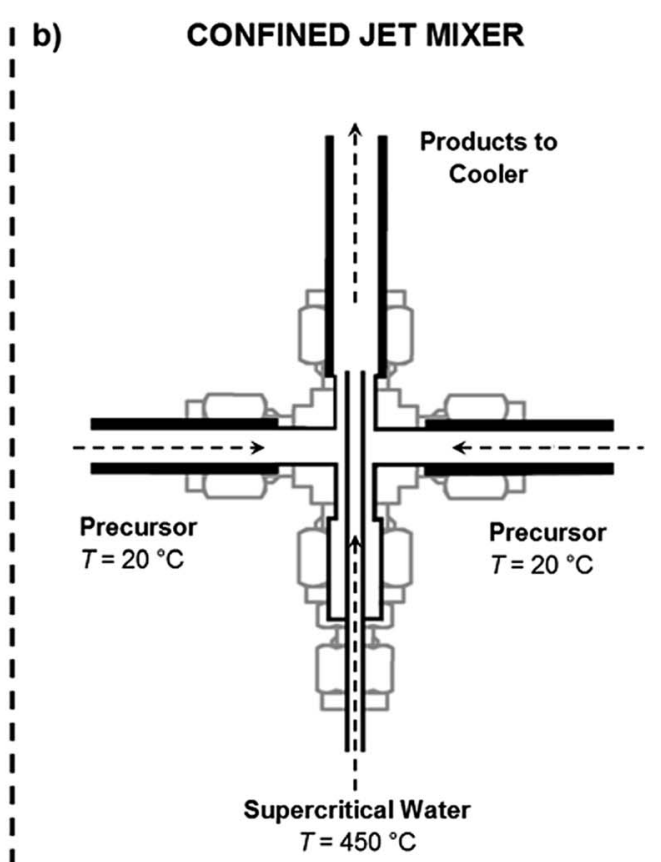

Fig. 1 (a) A schematic representing the continuous hydrothermal flow synthesis system described herein and (b) a schematic diagram representing the Confined Jet Mixer (CJM), in which the supercritical water enters from the bottom inner pipe. The ambient temperature aqueous metal salt feed (that has already been mixed with the base in flow) enters from both of the side arms and mixes with the sc-water just above the annulus of the inner pipe of the CJM. 
aqueous solution of zinc nitrate hexahydrate with either aluminium nitrate nonahydrate or gallium nitrate hydrate (with appropriate amount of the $\mathrm{Al}$ or Ga salt) at a flow rate of $40 \mathrm{~mL}$ $\min ^{-1}$ (pump $\mathrm{P}_{2}$ ) and (iii) an aqueous solution of potassium hydroxide at a flow rate of $40 \mathrm{~mL} \min ^{-1}$ (pump $\mathrm{P}_{3}$ ). The total concentration of the metal ions (not including the base) in the initial precursor feeds was maintained at $0.3 \mathrm{M}$, with the atomic ratio of the $[\mathrm{Zn}+\mathrm{Al}$ or Ga salts] being varied according to target dopant-loading for ZnO (see Tables S1 and S2 in the ESI†). The base concentration was maintained at $0.6 \mathrm{M}$ throughout. The experiments were conducted as follows; first the premixed metal precursor solution feed from pumps $\mathrm{P}_{2}$ and the base feed from $\mathrm{P}_{3}$ were mixed in a $1 / 4^{\prime \prime}$ stainless steel T-piece prior to mixing with the supercritical water flow in a Confined Jet Mixer (CJM) as shown in Fig. 1a. The design of the CJM is shown in Fig. 1b, and is detailed in previous publications of the authors..$^{27,29,39}$ The DI water feed from pump $\mathrm{P}_{1}$ was heated to $450{ }^{\circ} \mathrm{C}$ in flow using a $7 \mathrm{~kW}$ custom-built electrical heater. The reaction at this CJM mixing point resulted in the rapid crystallisation of nanoparticles at a calculated temperature of $325{ }^{\circ} \mathrm{C}$ (based on the properties of water and the temperatures and flow rates used). ${ }^{40}$ The particle-laden flow was then cooled to $c a$. $40{ }^{\circ} \mathrm{C}$ using a $1.5 \mathrm{~m}$ pipe-in-pipe heat exchanger, before passing through a back-pressure regulator (BPR), which maintained the pressure in the system. The aqueous nanoparticle slurry exiting the BPR was collected in a beaker and then cleaned by repeated centrifugation and washing with deionised water, until the conductivity of the supernatant was below $50 \mu \mathrm{S}$, as measured by a conductivity probe (Hanna Instruments, model HI98311). The concentrated slurry was then freeze-dried (Virtis Genesis $35 \mathrm{XL}$ ) by slowly heating the samples from $-60{ }^{\circ} \mathrm{C}$ to $25{ }^{\circ} \mathrm{C}$ over $24 \mathrm{~h}$ under vacuum of $<13 \mathrm{~Pa}$, which yielded free-flowing powders.

\section{Materials characterisation}

Powder X-ray diffraction (XRD) data were collected using a STOE Stadi P diffractometer (Mo-K $\alpha$ radiation, wavelength $=0.70932$ $\AA$ ) in transmission geometry for analysis. Data were collected over the $2 \theta$ range 5 to $30^{\circ}$ with a step size of $0.5^{\circ}$ and a count time of $10 \mathrm{~s}$ per step. Scherrer analysis was carried out on the (100), (002), and (102) peaks using eqn (1), where $\tau$ is the crystallite size (in $\mathrm{nm}$ ), $K$ is the dimensionless shape factor taken to be $0.9, \lambda$ is the wavelength of X-rays used (in nm), $\beta$ is the line broadening at half the maximum intensity for the peak in question (in radians), and $\theta$ is the Bragg angle (in degrees).

$$
\tau=\frac{K \lambda}{\beta \cos \theta}
$$

Transmission electron microscopy (TEM) was performed using a Jeol $200 \mathrm{kV}$ transmission electron microscope in imaging mode. Samples were dispersed in $\mathrm{MeOH}$ and drop coated onto a carbon-coated copper TEM grid purchased from Agar Scientific. Image analysis and particle size measurements were carried out using ImageJ ${ }^{\circledR}$ software. Energy dispersive $\mathrm{X}$-ray spectroscopy (EDS) analysis was carried out using an
Oxford Instruments X-Max ${ }^{\mathrm{N}}$ 80-T Silicon Drift Detector (SDD) fitted to the transmission electron microscope and processed using Aztec® software. X-ray photoelectron spectroscopy (XPS) was performed a Thermo Scientific K-alpha photoelectron spectrometer using monochromatic $\mathrm{Al}-\mathrm{K} \alpha$ radiation. Survey scans were collected in the range $\mathbf{0}-\mathbf{1 1 0 0} \mathrm{eV}$ (binding energy) at a pass energy of $160 \mathrm{eV}$. Higher resolution scans were recorded for the principal peaks of $\mathrm{Zn}(2 \mathrm{p}), \mathrm{Al}(2 \mathrm{p}), \mathrm{Ga}(3 \mathrm{~d}), \mathrm{O}(1 \mathrm{~s})$ and $\mathrm{C}$ (1s) at a pass energy of $50 \mathrm{eV}$. Peak positions were calibrated to carbon and plotted using the CasaXPS® software. Additional chemical analysis for $\mathrm{Zn}, \mathrm{Al}$ and $\mathrm{Ga}$ was performed by ICP-AES using dilute solutions of the samples dissolved in $1 \% \mathrm{HNO}_{3}$ (aq.). Analyses were carried out using a Varian 720 ICP-AES (axial configuration) equipped with an autosampler. Prior to sample analysis, calibrations were carried out using standards at concentrations of 2.5, 5.0, 7.5 and $10 \mathrm{ppm}$ for $\mathrm{Zn}$ and 0.25, $0.5,0.75$ and $1.0 \mathrm{ppm}$ for $\mathrm{Al}$ and $\mathrm{Ga}$. To assess the conductivity of the materials, the powders were pressed into $16 \mathrm{~mm}$ diameter green-coloured compacts of thickness $1.0 \mathrm{~mm}$ under a force of $50 \mathrm{kN}$ using a hydraulic press (Specac, Orpington, UK). The discs were then heat-treated under $5 \%$ hydrogen in nitrogen at
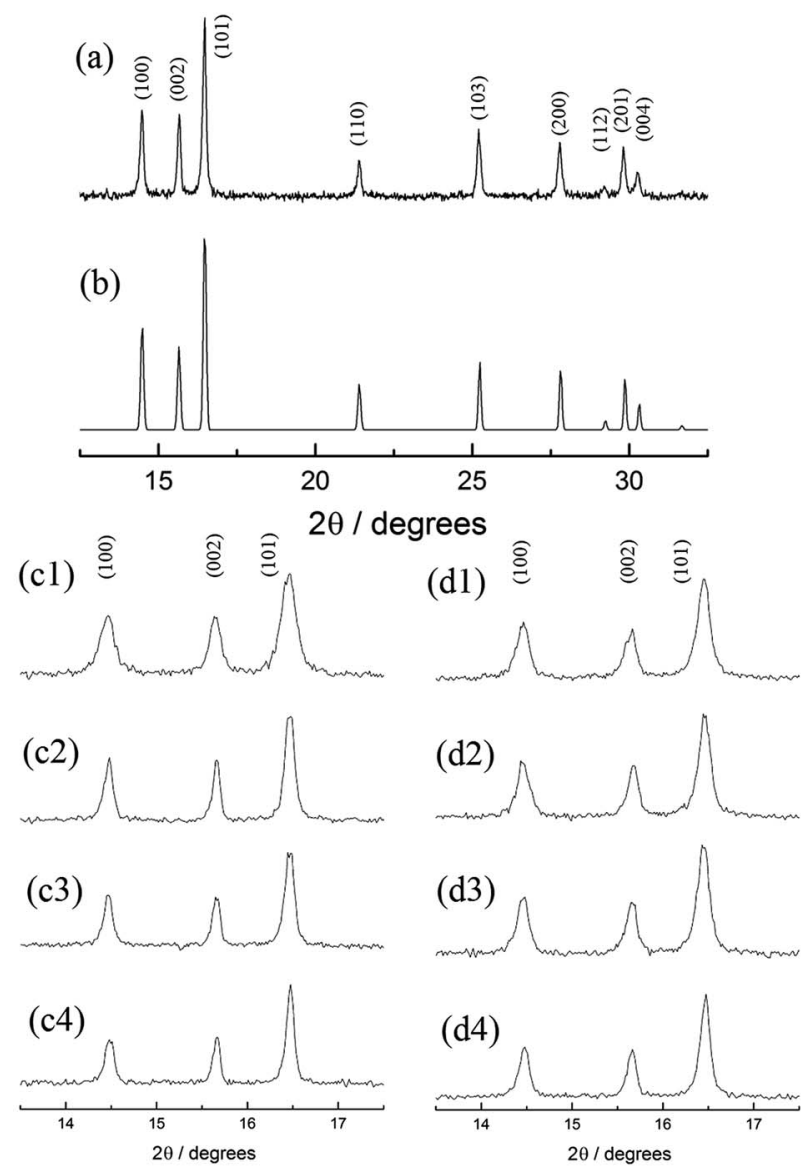

Fig. 2 XRD patterns for samples of each AZO and GZO. (a) shows a full pattern typical of the zinc oxides, in this case sample A1.0. (b) shows a standard $\mathrm{ZnO}$ pattern. ${ }^{40}$ (c1) and (d1) show peaks in the range 13.5 to $17.5^{\circ} 2 \theta$ for A2.5p and G2.5p, respectively (pilot scale materials), (c2-4) and (d2-4) show these same peaks for 5,3 , and 1 at $\%$ nominal dopant level for each AZO and GZO synthesised on the laboratory scale. 
$500{ }^{\circ} \mathrm{C}$ for 3 hours, after which a change in colour from green to blue was observed. Hall effect measurements were carried out using the Van der Pauw method to determine the bulk resistivity of the materials. In order to do this, four gold contacts were first sputtered onto the heat-treated discs, which were then subjected to an input current of $1 \mathrm{~mA}$ and a calibrated magnetic field of $0.58 \mathrm{~T}$ using the Van der Pauw probe. The transverse voltage was then measured. The measurement was repeated by reversing the direction of the magnetic field and the current. Resistivity measurements were made in triplicate for each pellet and the mean value and standard deviations were calculated.

\section{Results and discussion}

\section{Synthesis and characterisation of AZO and GZO nanopowders}

The following naming convention is in use in this work; letter A or $\mathrm{G}$ corresponds to the dopant being $\mathrm{Al}$ or $\mathrm{Ga}$, and the number following the letter represents the relative at\% of this metal ion in the precursor solution, such that A2.5 is the sample synthesised with 2.5 at\% $\mathrm{Al}$ in the precursor solution (with respect to 97.5 at\% $\mathrm{Zn}$ ). The suffix ' $\mathrm{p}$ ' is used to denote samples synthesised on the pilot scale, all others were synthesised on laboratory scale CHFS. In the initial lab-scale CHFS experiments, the concentration of the dopant in solution was in the range $0.5-6.0$ at\% (increments of 0.5 at\%) with respect to $\mathrm{Zn}$. Initially an off-white powder at low dopant levels, the colour of the products went from cream to pale yellow at high dopant levels. The GZO system powders were had more intense colour for each dopant level relative to the AZO system. The base concentration was consistently obtained at $\mathrm{pH} 7$, and the yield, though fluctuating from sample to sample, was consistently in the range 75 to $85 \%$.

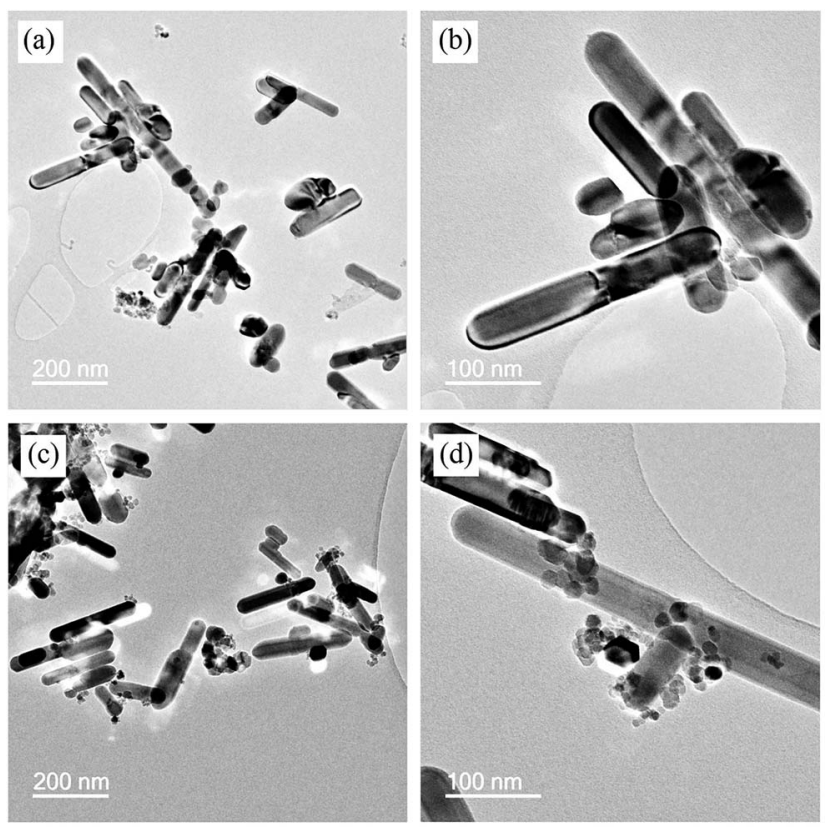

Fig. 3 TEM images of AZO. (a) and (b) are from A2.5; (c) and (d) are from A5.0.
Selected XRD patterns are shown in Fig. 2, with dopant levels in the precursor feeds of 1,3 , and 5 at\% for each AZO and GZO series, as well as a reference pattern for $\mathrm{ZnO}{ }^{41}$ The broad XRD peaks in the as-prepared materials was indicative of nanocrystallites; Scherrer peak analysis was performed on the diffractograms for the (100), (002), and (101) XRD peaks, the averages for which are collated in Tables S1 and S2 in the ESI. $\dagger$ Estimated crystallite size was observed to decrease with increasing dopant concentration in the precursor for each series; the sizes were calculated as 35 and $31 \mathrm{~nm}$ for samples A1.0 and A5.0, respectively, and 28 and $21 \mathrm{~nm}$ for samples G1.0 and G5.0, respectively.

TEM images revealed that the morphology of the aluminium-doped samples was a mixture of small $(<10 \mathrm{~nm})$ rounded particles and larger rod-like structures. On average these particles were $43 \pm 14 \mathrm{~nm}$ wide by $132 \pm 83 \mathrm{~nm}$ long (mean from 250 particles), an aspect ratio of 3.1 for sample A2.5 with 2.5 at\% Al dopant (see Fig. 3a and b).

Increasing the aluminium content in the precursor to 5.0 at\% (Fig. 3c and d), resulted in a decrease in average rod width to $38 \pm 17 \mathrm{~nm}$, but an increase in length to $187 \pm 92 \mathrm{~nm}$, corresponding to an increase in average aspect ratio to 4.9. This was consistent with the crystallite size as estimated by the Scherrer method; the crystallite size decreased with increasing $\mathrm{Al}$ at\%, as does the width of the rods as determined from TEM images.

BET analyses were conducted in order to understand the changes in surface area across the compositions. BET data suggested an increase in surface area from $19 \mathrm{~m}^{2} \mathrm{~g}^{-1}$ at 1 at\% $\mathrm{Al}$ up to $27 \mathrm{~m}^{2} \mathrm{~g}^{-1}$ for 5 at\% Al that was also consistent with the trends from TEM and Scherrer analyses, though the BET values were lower than expected. This was due to the difficulty in fully removing moisture from very high surface area $\mathrm{ZnO}$-based
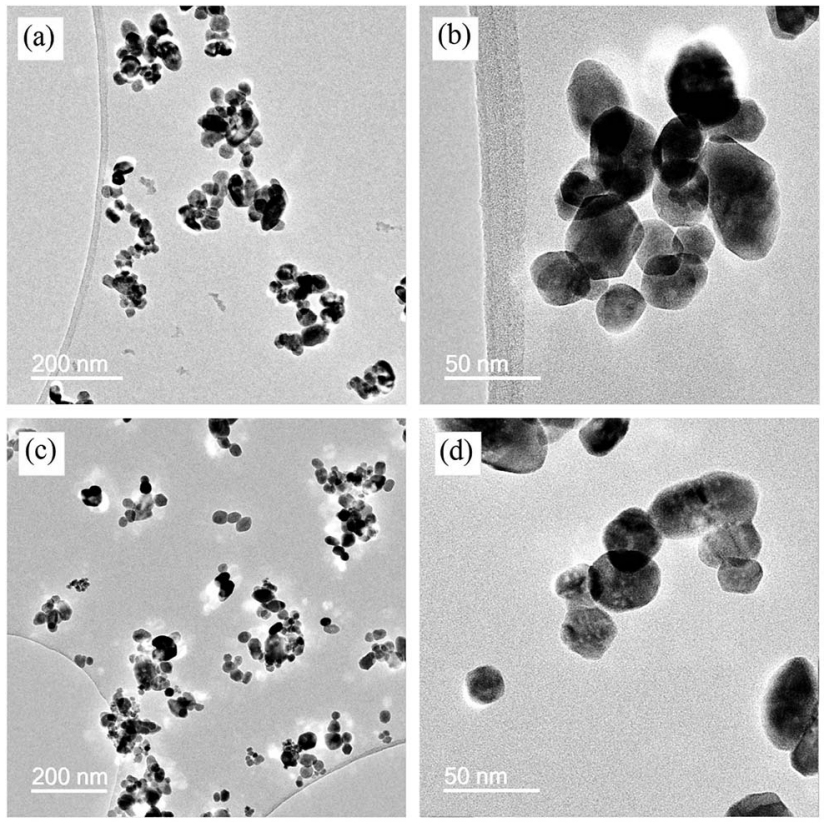

Fig. 4 TEM images of GZO; samples G2.5 ( $a$ and b) and G5.0 (c and d). 
samples prior to BET analysis, despite extensive degassing for 18 hours at $150{ }^{\circ} \mathrm{C}$ prior to BET measurements. XPS analyses revealed that only one type of $\mathrm{Al}$-species was present up to 5 at $\%$ $\mathrm{Al}$ (the highest nominal concentration tested), as shown in Fig. S1 in the ESI. $\dagger$ The binding energy peak at $73.2 \mathrm{eV}$ corresponds to $\mathrm{Al}^{3+}$ in coordination with oxygen. ${ }^{42} \mathrm{~A}$ single environment was observed for $\mathrm{Zn}$, with the $2 \mathrm{p}_{3 / 2}$ peak at $1021.5 \mathrm{eV}$ characteristic of $\mathrm{ZnO} .{ }^{43}$ ICP analyses suggested that the actual loadings of $\mathrm{Al}$ in the solids were 2.2 and 4.1 at\% for samples A2.5 and A5.0, respectively, indicating a dopant uptake of $>80 \%$ from the nominal compositions in the precursor solutions.

TEM images of the Ga-doped $\mathrm{ZnO}$ samples showed a rounded morphology with an aspect ratio close to 1 for the 2.5 at\% (Fig. 4a and b) and for the 5.0 at\% samples (Fig. 4c and d); the increase in $[\mathrm{Ga}]$ in the precursor resulted in a decrease of particle size from $44( \pm 8)$ to $36( \pm 9) \mathrm{nm}$, respectively. BET analyses of the Ga-doped $\mathrm{ZnO}$ samples demonstrated an increase in surface area with increasing Ga at\%, from $18 \mathrm{~m}^{2} \mathrm{~g}^{-1}$ at 1 at $\%$ Ga up to $31 \mathrm{~m}^{2} \mathrm{~g}^{-1}$ for 5 at\% Ga. Concurrently, as expected, the crystallite size as calculated using the Scherrer method, showed a general decrease in crystallite size with increasing dopant concentration.

TEM, XRD and BET data for the Ga-doped materials suggested that the effect of increasing [Ga] in the precursor solutions (and thus in the product) reached a plateau, such that the crystallite size and surface area did not dramatically change at $>3.5$ at\% Ga. XPS data suggested a possible reason for this; in the 2.5 at $\%$ Ga sample, the $3 \mathrm{~d}$ binding energy doublet revealed only a single gallium environment at $22.1 \mathrm{eV}$. This was consistent with the presence of $\mathrm{Ga}^{3+}$ and was attributed to $\mathrm{Ga}_{\mathrm{Zn}}$, gallium substituted into the Zn sites in ZnO. For sample G5.0, two sets of doublets were present in the spectrum; one at the same binding energy, representing a slightly higher at\% of Ga present in the surface structure, as well as a second peak at a lower binding energy of $19.7 \mathrm{eV}$. This second peak was representative of $\mathrm{Ga}_{2} \mathrm{O}_{3},{ }^{44}$ a separate species that appeared to have formed once a saturation point of $\mathrm{Ga}_{\mathrm{Zn}}$ was reached in the ZnO structure at ca. 3.0 (or 3.5) at\% gallium. ICP data indicated that the actual values of dopant Ga in the solid was 2.5 and 5.5

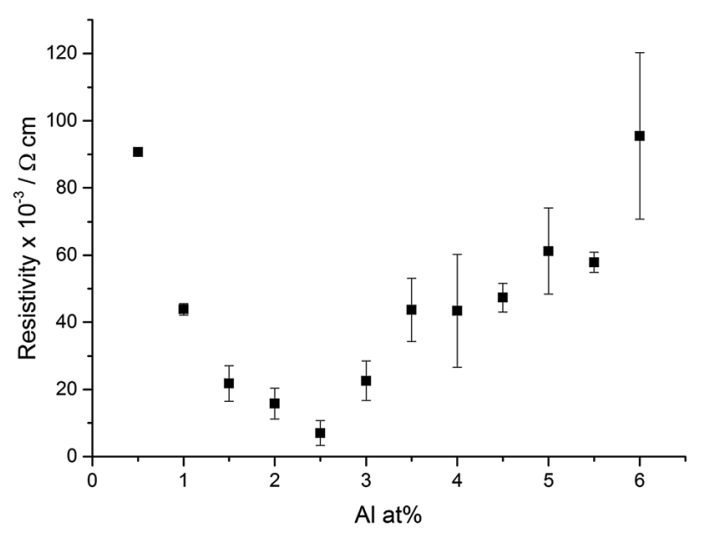

Fig. 5 Resistivity data for Al-doped $\mathrm{ZnO}$ with increasing dopant level. Error bars correspond to standard deviations in Hall probe data for 3 repeat measurements.

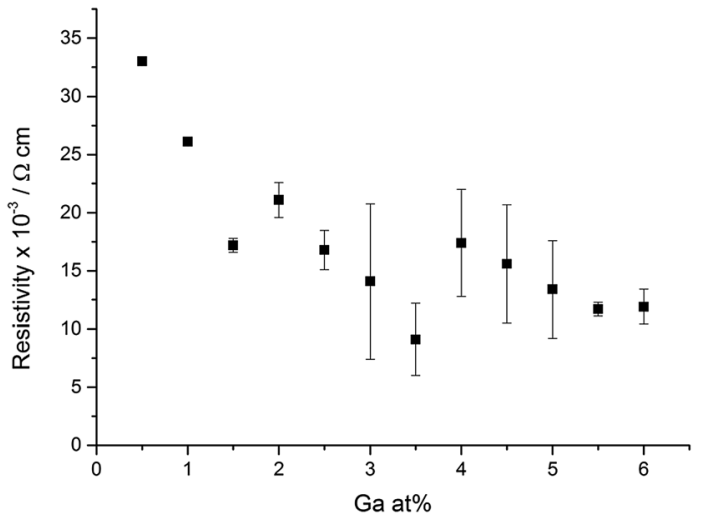

Fig. 6 Resistivity data for Ga-doped $\mathrm{ZnO}$ with increasing dopant level. Error bars correspond to standard deviations in Hall probe data for 3 repeat measurements.

at\% for samples G2.5 and G5.0, respectively, suggesting a slightly increased dopant uptake compared to the AZO samples.

\section{High-throughput screening of AZO and GZO nanomaterials}

Pressing of the powders into discs resulted in a colour change from off-white/yellow to green, the colour strength being dependant on the dopant concentration; after reducing heat treatment, the discs were all blue in colour. Similar colours were seen on both lab and pilot plant scales. The bulk resistivity of the sintered discs was determined by use of a Hall probe, by first sputter coating four gold contacts onto each disc. Hall effect measurements were carried out on these discs using the Van der Pauw method to determine the bulk resistivity of the materials. The measured resistivities as a function of the dopant content are summarised in Tables $\mathrm{S} 1$ and $\mathrm{S} 2 \uparrow$ for $\mathrm{AZO}$ and GZO, respectively, along with standard deviation in the results (shown graphically in Fig. 5 and 6). For AZO, this data clearly showed a minimum resistivity at 2.5 at\% $\mathrm{Al}$ (on the lab-scale CHFS), with an average value of $7.0 \pm 3.7 \times 10^{-3} \Omega \mathrm{cm}$. By comparison, ITO discs made by the lab scale CHFS process of the authors, showed optimum resistivity of $6.0 \pm 0.2 \times 10^{-3} \Omega$ $\mathrm{cm} .{ }^{30}$ For GZO, the resistivity decreased with increasing $\mathrm{Ga}$ content, reaching a minimum at 3.5 at\% Ga. However, further increase in gallium content did not improve the resistivity, which appeared to plateau thereafter. This is in keeping with the evidence from XPS, XRD and BET data, which suggested that there is a limit to the uptake of gallium into the $\mathrm{ZnO}$ structure.

The lowest mean resistivity value for 3.5 at\% GZO (sample G3.5), was $9.1 \times 10^{-3} \Omega \mathrm{cm}$. Both the optimum resistivity of AZO and that of GZO reported herein, are a significant improvement on the value of $4.6 \times 10^{-2} \Omega \mathrm{cm}$, as reported by the authors elsewhere for the corresponding co-doped system. ${ }^{36}$

\section{Pilot scale continuous hydrothermal synthesis of AZO and GZO}

Whilst the scale of the synthesis discussed thus far (rate of $60 \mathrm{~g}$ per hour) was suitable for high-throughput synthesis and 
screening of doped zinc oxide nanomaterials, production of such materials at still larger scales is critical for practical industrial applications. Previous reports by the authors have demonstrated the scale-up of the CHFS process for the synthesis of $\mathrm{ZnO}^{27}$ and $\mathrm{Ce}-\mathrm{Zn}$ oxide nanoparticles ${ }^{29}$ in a continuous supercritical water pilot plant. In both of these cases, it was shown that particles synthesised on the pilot scale were similar or indistinguishable from those synthesised on the smaller lab-scale process, based on TEM and BET data analysis. In the only report on the CHFS scale-up of a TCO, the authors previously showed that ITO could be synthesised with minimal change in material properties (size and resistivity) between laband pilot-scale CHFS processes. ${ }^{31}$

Herein for AZO, as the optimal dopant level was observed to be in the range $c a$. 2.0 to 2.5 at\% $\mathrm{Al}$ on the lab scale, dopant precursor solutions corresponding to 1.0, 2.0, 2.5, and 3.5 at $\%$ were selected for the pilot plant synthesis. For GZO, 2.0, 3.0, 3.5, and 4.5 at\% Ga were selected for scale-up; in both cases, these dopant levels should have given an indication of any trends in the data, i.e. if trends on lab scale were transferable to the pilot plant scale.

The as-prepared materials made on the pilot scale CHFS were phase-pure wurtzite structure as determined by XRD (Fig. 2c1 and d1; full patterns are included in Fig. S3 in the ESI, $\uparrow$ as well as representative TEM images). XPS analysis revealed that the uptake of aluminium in AZO was reduced on the pilot scale by almost half, whereas uptake of gallium in GZO was increased by a third compared to the lab scales. ICP analyses also indicated that the dopant uptake was $1.9 \mathrm{at} \% \mathrm{Al}$ in sample A2.5p and 1.7 at\% Ga in G2.5p. This showed quantitatively the decrease in dopant uptake when synthesising on the pilot scale, compared to 2.2 at\% $\mathrm{Al}$ and 2.5 at\% Ga in each A2.5 and G2.5, respectively, the lab-scale analogues.

The lower-than-expected dopant levels on the pilot plant can be understood when one considers the following: the residence time on the pilot plant $(<4 \mathrm{~s})$ is considerably less than the lab scale process under similar conditions $(>12 \mathrm{~s})$ and this is very important for precipitation of precursors that are highly soluble and that tend to nucleate and have a significant growth or maturation phase in flow. With less time to nucleate out from solution, the dopant elements were incorporated less effectively into the $\mathrm{ZnO}$ structure, and thus the materials made on the pilot scale demonstrated reduced uptake of the dopants compared to those synthesised on the lab scale. This could be rectified in the future by increasing the residence time on the pilot plant or by using more supersaturating conditions to drive the conversion of the $\mathrm{Zn}$ precursor.

To confirm the electrical performance of the pilot scale samples, compact pellets were again pressed and resistivity measurements taken. As detailed in Tables S1 and S2, $\uparrow$ the trend of conductivity as seen on the lab scale CHFS for both materials was carried forward to the pilot scale. The optimal resistivity obtained for AZO at 2.0 at\% on the pilot scale was $6.1 \times 10^{-2} \Omega \mathrm{cm}$, which is higher than the laboratory scale analogue of $1.6 \times 10^{-2} \Omega \mathrm{cm}$. This was believed to be due to increased grain boundary effects in the pellets due to reduced crystallite size of the pilot scale material, as well as differences in $\mathrm{Al}$ uptake between lab and pilot scale materials. The optimum GZO sample was at 4.5 at\% Ga, and this had a resistivity of $1.3 \times 10^{-2} \Omega \mathrm{cm}$, comparable to the analogous lab-scale material.

\section{Conclusions}

In summary, highly conductive AZO and GZO compacts were synthesised via continuous hydrothermal flow synthesis followed by heat-treatment of the products in a reducing atmosphere. The optimal compositions of each AZO and GZO on the lab scale were found to have 2.5 and 3.5 at\% dopant, respectively, with the optimal resistivities of $7.0 \times 10^{-3} \Omega \mathrm{cm}$ and $9.1 \times 10^{-3} \Omega \mathrm{cm}$, respectively. Pilot-scale CHFS was used for the attempted production of selected AZO and GZO samples at a rate of $8 \mathrm{~kg}$ per day. Analysis of these scaled-up materials showed that the particles were physically similar to the laboratory-scale process in terms of particle properties, with dopant uptake to the $\mathrm{ZnO}$ structure reduced on up-scaling of both AZO and GZO. Electrical characterisation revealed that pilot-scale AZO had lower conductivities than observed on the laboratory scale, but the optimum GZO sample was comparable, with optimal resistivity obtained of $1.3 \times 10^{-2} \Omega \mathrm{cm}$ for sample G4.5p. This work demonstrates the successful use of CHFS for the synthesis of sustainable TCO nanomaterials, in particular the rapid identification of optimal dopant compositions for both aluminium- and gallium-doped zinc oxides. Our optimal TCO materials are currently being investigated as sputtering targets to make thin films; results of these endeavours will be reported in due course.

\section{Acknowledgements}

The EPSRC is thanked for funding Award 1480036 (DPH), and the project with grant number EP/L017709/1 (JAD, CJC, IPP, PM). Prof. John McArthur is thanked for technical support with ICP measurements.

\section{References}

1 P. P. Edwards, A. Porch, M. O. Jones, D. V. Morgan and R. M. Perks, Dalton Trans., 2004, 17, 2995-3002.

2 K. Chopra, S. Major and D. Pandya, Thin Solid Films, 1983, 102, 1-46.

3 R. G. Gordon, MRS Bull., 2000, 25, 52-57.

4 S. Calnan and A. Tiwari, Thin Solid Films, 2010, 518, 18391849.

5 C. Granqvist, A. Azens, A. Hjelm, L. Kullman, G. Niklasson, D. Rönnow, M. S. Mattson, M. Veszelei and G. Vaivars, Sol. Energ., 1998, 63, 199-216.

6 J.-W. Park, G.-H. Lee, Y. Y Kwon, K.-W. Park, J. Lee, Y. W. Jin, Y.-C. Nah and H. Kim, Org. Electron., 2014, 15, 2178-2183.

7 T. Minami, Semicond. Sci. Technol., 2005, 20, S35.

8 R. B. H. Tahar, T. Ban, Y. Ohya and Y. Takahashi, J. Appl. Phys., 1998, 83, 2631-2645.

9 A. Stadler, Materials, 2012, 5, 661-683. 
10 H.-R. An, C. Kim, S.-T. Oh and H.-J. Ahn, Ceram. Int., 2014, 40, 385-391.

11 D. S. Bhachu, S. Sathasivam, G. Sankar, D. O. Scanlon, G. Cibin, C. J. Carmalt, I. P. Parkin, G. W. Watson, S. M. Bawaked, A. Y. Obaid, S. Al-Thabaiti and S. N. Basahel, Adv. Funct. Mater., 2014, 24, 5075-5085.

12 K. Ellmer, Nat. Photonics, 2012, 6, 809-817.

13 V. Sittinger, F. Ruske, W. Werner, C. Jacobs, B. Szyszka and D. Christie, Thin Solid Films, 2008, 516, 5847-5859.

14 S. Chen, N. Noor, I. P. Parkin and R. Binions, J. Mater. Chem. A, 2014, 2, 17174-17182.

15 F. Pattini, F. Annoni, F. Bissoli, M. Bronzoni, J. P. Garcia, E. Gilioli and S. Rampino, Thin Solid Films, 2015, 582, 317322 .

16 D. Kurita, S. Ohta, H. Ohta and K. Koumoto, J. Appl. Phys., 2006, 100, 096105-1.

17 G. Bühler, D. Thölmann and C. Feldmann, Adv. Mater., 2007, 19, 2224-2227.

18 M.-S. Hwang, B.-Y. Jeong, J. Moon, S.-K. Chun and J. Kim, Mater. Sci. Eng., B, 2011, 176, 1128-1131.

19 N. Al-Dahoudi and M. A. Aegerter, J. Sol-Gel Sci. Technol., 2003, 26, 693-697.

20 R. A. Street, W. Wong, S. Ready, M. Chabinye, A. Arias, S. Limb, A. Salleo and R. Lujan, Mater. Today, 2006, 9, 32-37.

21 J.-S. Lee and S.-C. Choi, J. Eur. Ceram. Soc., 2005, 25, 33073314.

22 T. Sasaki, Y. Endo, M. Nakaya, K. Kanie, A. Nagatomi, K. Tanoue, R. Nakamura and A. Muramatsu, J. Mater. Chem., 2010, 20, 8153-8157.

23 D. Ito, K. Masuko, B. A. Weintraub, L. C. McKenzie and J. E. Hutchison, J. Nanopart. Res., 2012, 14, 1-7.

24 B. G. DeLacy, S. Lacey, D. Zhang, E. Valdes and K. Hoang, Mater. Lett., 2014, 117, 108-111.

25 J. Ba, D. Fattakhova Rohlfing, A. Feldhoff, T. Brezesinski, I. Djerdj, M. Wark and M. Niederberger, Chem. Mater., 2006, 18, 2848-2854.

26 E. Hammarberg, A. Prodi-Schwab and C. Feldmann, Thin Solid Films, 2008, 516, 7437-7442.

27 R. I. Gruar, C. J. Tighe and J. A. Darr, Ind. Eng. Chem. Res., 2013, 52, 5270-5281.
28 J. Darr, C. J. Tighe and R. Gruar, US Pat. 9,192,901, 2015.

29 C. J. Tighe, R. Q. Cabrera, R. I. Gruar and J. A. Darr, Ind. Eng. Chem. Res., 2013, 52, 5522-5528.

30 X. Weng, J. K. Cockcroft, G. Hyett, M. Vickers, P. Boldrin, C. C. Tang, S. P. Thompson, J. E. Parker, J. C. Knowles, I. Rehman, I. P. Parkin, J. R. G. Evans and J. A. Darr, J. Comb. Chem., 2009, 11, 829-834.

31 P. Marchand, N. M. Makwana, C. J. Tighe, R. I. Gruar, I. P. Parkin, C. J. Carmalt and J. A. Darr, ACS Comb. Sci., 2016, 18, 130-137.

32 J. B. M. Goodall, D. Illsley, R. Lines, N. M. Makwana and J. A. Darr, ACS Comb. Sci., 2015, 17, 100-112.

33 R. Quesada-Cabrera, X. Weng, G. Hyett, R. J. H. Clark, X. Z. Wang and J. A. Darr, ACS Comb. Sci., 2013, 15, 458-463.

34 T. Lin, S. Kellici, K. Gong, K. Thompson, J. R. Evans, X. Wang and J. A. Darr, J. Comb. Chem., 2010, 12, 383-392.

35 S. J. Alexander, T. Lin, D. J. Brett, J. R. Evans, G. Cibin, A. Dent, G. Sankar and J. A. Darr, Solid State Ionics, 2012, 225, 176-181.

36 D. P. Howard, P. Marchand, T. Gordon and J. A. Darr, J. Nanosci. Nanotechnol., 2016, 16, 10166-10171.

37 L. Shi, A. Naik, J. B. M. Goodall, C. J. Tighe, R. I. Gruar, R. Binions, I. P. Parkin and J. A. Darr, Langmuir, 2013, 29, 10603-10609.

38 X. Weng, J. Zhang, Z. Wu, Y. Liu, H. Wang and J. A. Darr, Green Chem., 2011, 13, 850.

39 C. Y. Ma, X. Z. Wang, C. J. Tighe and J. A. Darr, Adv. Control Chem. Processes, Proc. Vol. IFAC Symp., 2012, 8, 874-879.

40 W. Wagner and A. Pruß, J. Phys. Chem. Ref. Data, 2002, 31, 387-535.

41 H. Schulz and K. Thiemann, Solid State Commun., 1979, 32, 783-785.

42 J. Waldrop, G. Sullivan, R. Grant, E. Kraut and W. Harrison, J. Vac. Sci. Technol., B: Microelectron. Nanometer Struct.Process., Meas., Phenom., 1992, 10, 1773-1778.

43 V. Nefedov, M. Firsov and I. Shaplygin, J. Electron Spectrosc. Relat. Phenom., 1982, 26, 65-78.

44 C. Surdu-Bob, S. Saied and J. Sullivan, Appl. Surf. Sci., 2001, 183, 126-136. 